\title{
Indicateurs alternatifs au PIB : Au-delà des nombres
}

\author{
L'Épargne nette ajustée en question*
}

Géraldine Thiry

\begin{abstract}
Après avoir dominé pendant plus de soixante ans les agendas politiques des pays occidentaux, l'objectif de croissance économique et son indicateur-phare, le produit intérieur brut (PIB), sont aujourd'hui largement questionnés. De nombreux indicateurs alternatifs sont suggérés et mis en débat. Ces initiatives sont une opportunité de changement : ce que nous mesurons influence ce que nous faisons. Mais il s'agit d'aller un pas plus loin, en montrant que la manière dont nous mesurons est tout aussi déterminante : les indicateurs sont porteurs de conceptions axiologiques et normatives, liées à leur mode de construction, dont la mise en lumière est cruciale là où les indicateurs ont une importance politique croissante. Notre analyse de l'Épargne nette ajustée (ENA, indicateur de soutenabilité de la Banque Mondiale) illustre les enjeux normatifs d'un choix de mesure, souvent occulté derrière des considérations techniques : nous montrons que l'ENA porte une conception singulière et discutable de 'soutenabilité' (dans ses aspects écologique et humain).

Mots-clés: au-delà du PIB, sociologie de la quantification, épistémologie des statistiques, indicateurs, Épargne nette ajustée, développement durable.

After sixty years of predominance in the western countries, both the objective of economic growth and its core measure, the Gross Domestic Product (GDP), have been questioned. Numerous alternative indicators have been suggested. These new indicators potentially constitute a great opportunity for change: what we measure affects what we do. We go a step further, claiming that the way we measure it is just as crucial: indicators intrinsically carry axiological and normative conceptions, embedded in the specific way

\footnotetext{
* Ce texte constitue une synthèse en français d'un article plus détaillé en anglais: THIRY G., CASSIERS I.: «Alternative Indicators to GDP : Values behind Numbers. Adjusted Net Savings in Question » à paraitre dans Applied Research in Quality of Life en 2010 et publié sous le même titre comme Discussion Paper de l'IRES n 2010-018, disponible sur http://sites.uclouvain.be/econ/DP/IRES/2010018.pdf
} 
they are built. As indicators are increasingly being used for shaping political ends, light should be shed on these underlying conceptions. Our analysis of the Adjusted Net Savings (ANS, the sustainability indicator of the World Bank) attempts to illustrate these normative underpinnings, often obscured by technical concerns: we show that the ANS carries a singular conception of 'sustainability' (in its human and ecological aspects).

Key words: beyond GDP, sociology of quantification, epistemology of statistics, indicators, Adjusted Net Savings, Sustainable Development.

\section{Introduction}

Depuis plus de soixante ans, la croissance économique, évaluée à l'aide du produit intérieur brut (PIB), constitue un objectif central dans l'élaboration des politiques socioéconomiques en Occident. Créés au sortir de la Seconde Guerre Mondiale, les systèmes de comptabilité nationale - dont le PIB est l'indicateur-phare - ont été façonnés aux fins de répondre à un urgent besoin de reconstruction et de bâtir une nouvelle image de puissance nationale (FOURQUET 1980). Depuis lors, le PIB s'est érigé en balise incontournable dans la prise décision. Au long des Trente Glorieuses (19451975), l'objectif de croissance s'est trouvé légitimé par une large acceptation du lien entre progrès social et accroissement de la production faisant l'objet d'un échange marchand. Cette vision du progrès s'inscrivait dans des pactes sociaux qui institutionnalisaient le partage systématique des fruits de la croissance entre travail et capital.

Aujourd'hui toutefois, les impacts environnementaux et sociaux de soixante ans de croissance ont conduit à un tournant historique: il n'apparaît plus possible de maintenir, en l'état, la croissance économique comme finalité sociétale ultime. Il n'est plus pertinent non plus de conserver le PIB comme référence majeure dans les politiques socioéconomiques. Le PIB n'a pas été en mesure de saisir correctement des enjeux liés à la qualité et à la soutenabilité des modes de vie des personnes et des sociétés. Il n'a pas non plus été apte à mettre en garde contre la montée de différents facteurs de crise, comme l'a montré l'éclatement des récentes crises financière et économique.

De nombreux débats ont émergé sur les possibilités d'aller au-delà de cet indicateurclé, mettant en présence des acteurs d'horizons variés: citoyens, politiques, organisations non-gouvernementales, institutions économiques internationales et académiques ${ }^{1}$. De ces débats ont émanés des alternatives et des compléments au PIB ${ }^{2}$.

1 Parmi les initiatives principales, mentionnons :

- Le 3ème Forum mondial de l'OCDE, "Charting Progress, Building Visions, Improving lives ", Octobre 2009, Busan, Corée du Sud; 
L'intérêt croissant pour la recherche de nouveaux indicateurs, amplifié au cours des quinze dernières années, et le fait que ceux-ci soient pris en charge par de puissantes institutions constituent un phénomène significatif: les nouveaux indicateurs de " progrès » sont amenés à jouer un rôle croissant dans la sphère publique. D’une part, en catégorisant le réel, ils forment d'importantes balises politiques à un moment où les mécanismes de gouvernance par «soft-law» prennent un essor inédit. D’autre part, et plus fondamentalement, les indicateurs de " progrès », par leur mode de construction et les catégories qu'ils opèrent, participent de la définition même du "progrès ». Le choix d'un indicateur (ou d'un ensemble d'indicateurs) est intrinsèquement porteur de valeurs et de visions du monde multiples ${ }^{3}$.

Les débats actuels ont donc la singularité de mêler questions de finalités (qu'est-ce qu'une société désirable? qu'est-ce que le progrès d'une société ?) et questions de méthode (comment mesurer le progrès d'une société ?). Alors que les enjeux de finalités requièrent la légitimité d'un débat transparent et ouvert, les questions techniques de mesure apparaissent souvent comme l'apanage naturel des experts. Or, les questions de finalités et les questions de méthode étant difficilement inextricables, le risque est grand de voir les premières absorbées par les secondes, attribuant aux experts le pouvoir de choisir une mesure de "progrès » et, indirectement, de décider de ce que le "progrès » serait et devrait être en catégorisant ce qui en ressort et ce qui en est exclu. Afin d'éviter un tel risque de déficit démocratique, il importe de décrypter de manière systématique les conceptions et catégories axiologiques et normatives que portent intrinsèquement les outils de mesure quantitatifs.

Il est de plus en plus largement accepté que tout choix de mesure est implicitement normatif. «Ce que l'on mesure a une incidence sur ce que l'on fait ; or, si les mesures sont défectueuses, les décisions peuvent être inadaptées» (STIGLITZ et al. 2009, p.7). Allant un pas plus loin, nous pensons que la manière dont on mesure est tout aussi

- La «Commission sur la mesure des performances économiques et du progrès social » (dont le rapport à été publié le 14 septembre 2009, à Paris, France)

- La conférence internationale du parlement Européen (en collaboration avec l'OCDE et le WWF), « Au-delà du PIB », Novembre 2007, Bruxelles, Belgique.

- Le 2ème Forum de l'OCDE, «Measuring and Fostering the Progress of Societies », Juin 2007, Istanbul, Turquie.

2 Pour un bon aperçu des alternatives actuellement proposées, se référer à GADREY \& JANYCATRICE (2007).

3 Notons que la normativité inhérente aux choix de mesure n'est pas l'apanage des débats sur une alternative au PIB. Elle est étudiée dans l'ensemble des champs du social et fait l'objet d'une sociologie de la quantification dont Alain Desrosières constitue l'un des pionniers: "La quantification, entendue comme l'ensemble formé des conventions socialement admises et des opérations de mesure, crée une nouvelle façon de penser, de représenter, d'exprimer le monde et d'agir sur lui» (DESROSIÈRES 2008, p.11). 
cruciale dans la construction de la représentation du «progrès » et dans l'orientation des décisions et des comportements collectifs et individuels. Pourtant, les fondements et les impacts de ce que nous mesurons et de la manière dont nous le mesurons sont trop souvent occultés derrière des considérations d'ordre méthodologique. En passant ici en revue un indicateur d'amont en aval, de son cadre de référence théorique à ses sousdimensions, il s'agira de mettre en lumière les valeurs implicites sous-jacentes à une mesure quantitative, trop souvent présentée comme neutre ${ }^{4}$.

L'indicateur choisi ici est l'Épargne nette ajustée (ENA, aussi connue comme Épargne véritable) $)^{5}$. Cet indicateur de soutenabilité jouit d'une large visibilité. Il a été développé par la Banque Mondiale et est estimé pour 190 pays sur la période 19702006 ${ }^{6}$. L’ENA est prônée par de nombreux économistes pour être ancrée dans un cadre théorique et conceptuel cohérent. Par ailleurs, l'ENA s'est vu explicitement assigner un rôle de balise politique : «The [adjusted net savings]seeks to provide national-level decision makers with a clear, relatively simple indicator of how sustainable their country's investment policies are» (BOLT et al. 2002, p.4). De plus, le rapport de la Commission sur la mesure de la performance économique et du progrès social, a suggéré que l'ENA apparaisse (conditionnellement à quelques modifications) dans un set restreint d'indicateurs de progrès social (STIGLITZ et al. 2009). Les Nations Unies travaillent également à intégrer l'ENA dans leurs lignes directrices officielles (UNSD 1993), élargissant de ce fait l'impact potentiel de cet indicateur sur la décision politique à un niveau global (FERREIRA \& VINCENT 2005). Dès lors que l'ENA est amenée à influencer les conclusions de politique, notamment au niveau de la Banque Mondiale, sa portée internationale appelle à ce que les aspects normatifs inhérents à son mode de construction soient explicités. Cet exercice de transparence constitue, comme l'ont montré les membres du FAIR ${ }^{7}$, une condition nécessaire (bien que non suffisante) à la légitimité de l'ENA comme outil politique.

La déconstruction de l'ENA a été effectuée en profondeur dans Thiry et Cassiers (2010). Le présent article a pour objet de synthétiser cette décomposition et d'en faire ressortir les principaux axes en termes d'analyse normative. Il s'agira de montrer en quoi « indicateurs» et «catégories» (sociale, politique et cognitive) sont

\footnotetext{
${ }^{4}$ Chacun des enjeux normatifs présents ici mérite une analyse plus approfondie. Une telle analyse dépasserait l'ambition de cet article, dont l'objectif est bien de tracer un aperçu synthétique des questions normatives inhérentes aux processus de quantification.

5 En anglais : Adjusted Net Savings (épargne nette ajustée) ou Genuine Savings (épargne véritable).

${ }^{6}$ Les estimations de l'ENA sont téléchargeables sur http://go.worldbank.org/VLJHBLZP71

7 FAIR, Forum pour d'autres indicateurs de richesse. Merci à Jean Gadrey, Florence Jany-Catrice, Dominique Méda, Pascal Petit et Patrick Viveret pour leurs riches réflexions sur l'ENA. Merci également à Bernard Swartenbroeckx et Tanguy Isaac pour leurs stimulantes idées.
} 
inextricablement liés : les premiers reposent sur les secondes tout en contribuant au déploiement de nouvelles catégories. Nous rappelons d'abord comment l'ENA est construite. La deuxième section aborde les implications normatives du cadre de référence théorique de l'ENA. La section 3 discute l'hypothèse de substituabilité entre capital produit, capital humain et capital naturel. Les sections 4, 5 et 6 passent en revue quelques aspects normatifs sous-jacents à chacune des sous-dimensions de l'ENA. La section 4 discute la quantification de la dimension « humaine ». La section 5 questionne les modalités de quantification des ressources naturelles et leur imputation. La section 6 aborde le calcul des dommages liés aux émissions de dioxyde de carbone. La section 7 conclut.

\section{L’Épargne Nette Ajustée: un indicateur de « soutenabilité »}

L'ENA est un indicateur de soutenabilité. Elle a pour but de rendre compte de la création/destruction nette de richesse nationale endéans une année. Dans l'ENA, le concept de richesse est élargi pour inclure, outre le capital produit (seul capital présent dans la comptabilité nationale), les ressources naturelles, la qualité de l'environnement et le capital humain. Dérivée de la mesure traditionnelle d'épargne nationale brute (part du revenu national qui n'est pas consommée pendant l'année), l'ENA opère quatre types d'ajustements (graphique 1). Premièrement, une estimation de la consommation de capital fixe est déduite, pour obtenir l'épargne nationale nette : c'est l'amortissement. Ensuite, les dépenses courantes d'éducation (livres et salaires des professeurs principalement) sont ajoutées pour refléter l'investissement en capital humain. Troisièmement, les estimations de l'épuisement de certaines ressources naturelles sont déduites afin de représenter le déclin de leur valeur associé à leur extraction et/ou à leur récolte. Enfin, les dommages liés à la pollution globale par le dioxyde de carbone sont soustraits (BOLT et al. 2002; STIGLITZ et al. 2009).

\section{Graphique 1: Épargne nette ajustée (ENA)}

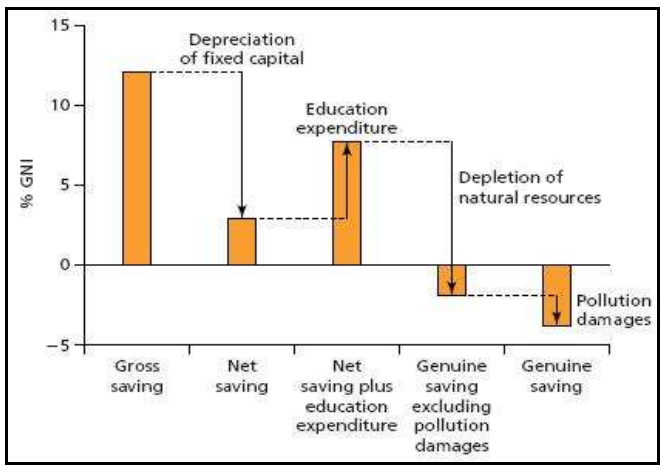

Source: Banque Mondiale 
Toutes ces variations de capitaux sont exprimées dans une unité de compte commune, en pourcentage du revenu national brut (RNB) et sont sommées.

$$
E N A=\frac{E N B-D_{h}+D C E-\sum R_{n, i}-D C}{R N B}
$$

Où

ENA = Épargne nette ajustée (en pourcentage du RNB)

$\mathrm{ENB}=$ Épargne nationale brute

$\mathrm{D}_{\mathrm{h}}=$ Dépréciation du capital produit

DCE $=$ Dépenses courantes d'éducation (hors capital fixe)

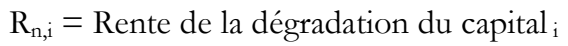

$\mathrm{DC}=$ Dommages résultant des émissions de dioxyde de carbone

$\mathrm{RNB}=$ Revenu national brut

Étant donné que cet indicateur représente la variation de la richesse sur une année, une ENA négative (une diminution nette de la richesse) signifie qu'il ne sera pas possible de maintenir les niveaux actuels de consommation dans le futur ou, en d'autres termes, qu'il y a surconsommation et sous-investissement par rapport à l'objectif de « soutenabilité ».

\section{L’ENA : un cadre théorique néo-classique}

Le passage en revue du cadre théorique d'un indicateur revêt un intérêt significatif : ce cadre comporte, dans son axiomatique, des éléments normatifs, catégories de représentation et visions du monde discutables. Dès lors que l'indicateur est voué à être utilisé ou compris en termes de finalités de société, ces catégories et visions du monde doivent être mises en lumière. Cette section explore la conception de soutenabilité sous-jacente à l'ENA (2.1). Elle discute ensuite, de manière critique, les implications d'une telle conception (2.2.).

\subsection{La conception de « soutenabilité » sous-jacente à l'ENA}

Dès ses premières formulations ${ }^{8}$, l'ENA, issue de la littérature sur la comptabilité verte et le bien-être social ${ }^{9}$, a l'ambition de fournir un indicateur de soutenabilité: une

8 Les premières formulations de l'ENA (épargne brute ajustée aux variations positives ou négatives de différents actifs), comme mesure améliorant les mesures traditionnelles d'épargne, 
mesure qui prendrait en compte l'épuisement des ressources vivantes et non-vivantes, la dégradation de l'environnement et l'investissement en capital humain (PEARCE \& ATKINSON 1993). Ce nouveau type de mesure enrichirait la comptabilité nationale en offrant les possibilités d'une meilleure conceptualisation de la soutenabilité.

Quelles que soient les diverses modalités de construction de l'ENA, celle-ci est communément construite, à la suite de Hartwick (1990), sur base d'un problème d'optimisation inter-temporel. La richesse est définie comme la valeur présente actualisée du bien-être, entendu comme l'utilité d'un agent représentatif. Le bien-être, représenté par la fonction d'utilité de l'agent représentatif, dépend et de deux éléments : la consommation et les services environnementaux ${ }^{10}$. Cette richesse doit être maximisée à travers le temps, sous la contrainte d'une fonction de production qui combine des actifs produits, naturels et humains (HAMILTON, ATKINSON \& PEARCE 1997). L'ENA résulte de la résolution de ce problème d'optimisation et est identifiée comme l'investissement en actifs produit et humain, desquels la valeur de l'épuisement des ressources naturelles et les dommages de la pollution sont déduits, qui assure la maximisation du bien-être à travers le temps. Cette conception de la soutenabilité s'inscrit dans le sillage de Pezzey (1989) qui définit la soutenabilité comme la valeur non décroissante de l'utilité à travers le temps.

\subsection{Implications normatives du cadre théorique de l'ENA}

La conception de soutenabilité inhérente au cadre théorique et conceptuel de l'ENA, qui associe intrinsèquement maintien de l'utilité et maintien des actifs à travers le temps, soulève plusieurs questions.

Premièrement, en dédiant à l'agent représentatif une place centrale (la soutenabilité est la maximisation de la fonction d'utilité de l'agent représentatif à travers le temps), l'ENA est ancrée dans un individualisme méthodologique qui empêche de penser l'évolution du bien-être collectif. Certes, la Banque Mondiale considère que le capital intangible (capital humain et qualité des institutions formelles et informelles) est prépondérant dans une conception élargie de la richesse: «Intangible assets (...)

apparaît dans Pearce et Atkinson (1993), Hamilton (1993), Hamilton (1994) et Hamilton, Pearce and Atkinson (1997). Hamilton et Clemens (1999) fournissent une base plus formelle à l'ENA; les auteurs y présentent plus rigoureusement les ajustements nécessaires aux mesures d'épargne traditionnelles pour créer un indicateur plus compréhensif.

9 Weitzman (1976), Dasgupta et Heal (1979), Hartwick (1990) et Mäler (1991) constituent des contributions séminales dans ce champ.

${ }^{10}$ Ces "services" affectent positivement l'utilité dans le cas des ressources naturelles (comme par exemple, les espaces verts et les forêts) tandis qu'ils affectent négativement l'utilité en cas de pollution. 
includes social capital, that is, the trust among people in a society and their ability to work together for a common purpose » (BANQUE MONDIALE 2006, p.18). Or, alors qu'une telle conception inclut des éléments de nature collective, le fait que l'ENA soit conçue comme un agrégat (somme nette des investissements dans les différents actifs de la richesse élargie) ne laisse pas d'espace à la prise en compte de la nature collective des capitaux social et institutionnel, dans leur aspect interactionnel notamment. Plus fondamentalement, cet individualisme méthodologique ne permet pas de considérer les enjeux de nature distributionnelle, dont les inégalités de revenus constituent un élément central, comme affectant la soutenabilité. Est-ce à dire que les inégalités ne jouent aucun rôle dans la soutenabilité d'une société ? Il est pourtant possible d'inclure les inégalités : des mesures alternatives de soutenabilité, dont le cadre conceptuel est assez proche de celui de l'ENA, suggèrent de pondérer négativement le degré d'inégalités de revenus. C'est le cas, notamment, de l'Indice de Bien-être Economique Durable (IBED, DAly \& CoBb 1989) et de l'Indice de Progrès Véritable (IPV, TAlberTh, CobB \& SLATTERY 2006).

Deuxièmement, dans un cadre théorique où l'environnement n'est pris en compte qu'à travers la fonction d'utilité d'un agent représentatif, la nature est envisagée uniquement au prisme des biens et services que l'on peut en extraire. De ce cadre théorique, la valeur d'existence de la nature est exclue, c'est-à-dire la valeur qui lui est accordée indépendamment de tout acte de production et de consommation. (EVERETT \& WiLKS 1997; FALCONI 1999). Cette approche de la soutenabilité est porteuse d'une vision instrumentale de la relation de l'humain à la nature. Certes, cette instrumentalité ne constitue pas un problème en soi puisque la maitrise de la nature par l'humain implique tant le risque d'une dégradation de la nature que la possibilité d'une contribution à son émancipation. Cette instrumentalité devient en revanche problématique quand elle se fonde, comme dans l'ENA, sur des hypothèses de préférences temporelles qui favorisent les logiques de court-terme (promptes à l'instrumentalisation de la nature à des fins de production/consommation) à celles de long-terme (favorisant la préservation de la nature pour pérenniser ses services).

Une troisième question, plus fondamentale, émerge du flou conceptuel qui entoure la définition même de ce qu'est la soutenabilité. Si les préférences temporelles sont telles que le court-terme est préféré au long-terme, on peut imaginer que la pondération implicite de la consommation dans la fonction d'utilité devienne plus importante que les services environnementaux. Dans un tel cas, rien n'assure que le maintien de l'utilité à travers le temps ne coïncide avec le maintien des actifs (élargis aux actifs naturel et humain). Cette question mérite réflexion. Elle s'avère pour l'heure peu développée dans la littérature de la comptabilité verte, comme en témoigne l'imprécision conceptuelle qui définit par moments la soutenabilité comme valeur non-décroissante de l'utilité à travers le temps (PEZZEY 1989), faisant de la soutenabilité une question de nature subjective, et parfois comme valeur non-décroissante des actifs (HAMILTON, ATKINSON \& PEARCE 1997), la soutenabilité étant une question de nature physique ou 
technique. Investiguer ce lien en apparence inextricable entre l'utilité et les actifs devrait permettre de mettre en lumière les déterminants et les finalités de la soutenabilité comme objectif politique et de mieux définir les limites et les orientations de la relations humain-nature.

En somme, tandis que l'ENA est prônée par de nombreux économistes pour son ancrage théorique cohérent, aucune considération explicite n'est faite sur les conceptions normatives inhérentes à son cadre théorique et les conséquences qu'un tel cadre génère. Il s'avère pourtant que celui-ci façonne une conception singulière de la soutenabilité : l'ENA est ancrée dans une individualisme méthodologique empêchant de penser les déterminants collectifs du bien-être à travers le temps et les questions de redistribution; cet indicateur porte dans ses fondements une vision très instrumentale et anthropocentrée du rapport humain-nature ; enfin, le cadre de référence théorique fait planer une large imprécision quant à la nature de la soutenabilité, visant tantôt des subjectivités, tantôt l'accumulation d'actifs.

Le passage du cadre théorique aux dimensions constitutives de l'ENA montrera en quoi les choix techniques de construction de cet indicateurs nourrissent et amplifient les conceptions néoclassiques mises en lumière dans cette section.

\section{Substituabilité: « Soutenabilité faible »}

En sommant des types de capital hétérogènes ramenés dans une unité de compte commune, l'ENA suppose, dans une certaine mesure, que ces capitaux sont substituables les uns aux autres. Selon cette approche, si le Brésil détruit la forêt amazonienne pour en vendre le bois et investit le chiffre d'affaires de cette vente en dépenses d'éducation, l'ENA restera inchangée, voire même augmentera. À l'aune de cet indicateur, le Brésil serait donc sur une trajectoire «soutenable » (PILLARISETTI \& VAN DEN BERGH 2008), tandis que la perte des services globaux de la forêt amazonienne en matière d'absorption de dioxyde de carbone serait passée sous silence. Une telle hypothèse de substituabilité s'inscrit dans une approche dite de "soutenabilité faible ».

D’un point de vue normatif, cette conception «faible» de la soutenabilité a été largement contestée. L'ENA, aux fins d'enrichir la conception de richesse inhérente à la comptabilité nationale, comptabilise la nature comme un «capital» parmi d'autres. Ce type de comptabilisation a un effet performatif : dès lors que l'impact de l'humain sur la nature se voit octroyer un "poids » économique, il devient conceptuellement possible 
de réparer les externalités ${ }^{11}$ environnementales négatives de l'activité humaine par un investissement "bénéfique " par ailleurs (HOLLAND 1999). Le cadre heuristique que crée l'hypothèse de substituabilité n'inclut aucune idée de limites écologiques et s'oppose en ce sens à la soutenabilité «forte », dont la prise en compte des limitations physiques de la nature constitue le fondement.

À la critique de substituabilité, d'aucuns répondront que la mise en place d'un système de prix approprié est apte à réduire le degré de substituabilité entre capitaux, faisant de l'indicateur monétaire un bon indicateur de soutenabilité «forte». Théoriquement en effet, les prix devraient refléter la rareté des ressources : ils devraient donc tendre vers l'infini au fur et à mesure que les ressources s'épuisent. Il deviendrait dès lors de plus en plus difficile de compenser une perte de ressources naturelles par un investissement en capital (produit ou humain), rendant de facto ces capitaux de moins en moins substituables. Empiriquement toutefois, comme les sections 5 et 6 l'illustreront, les ressources naturelles (tout comme les dommages environnementaux) sont souvent sous-estimés, empêchant de ce fait de limiter le degré de substituabilité avec les capitaux humain et produit ${ }^{12}$.

Cette difficulté empirique met en doute la pertinence d'un indicateur monétaire pour quantifier la soutenabilité «forte ». À l'aune de cette difficulté, certains indicateurs, qui se sont d'emblée départis d'une comptabilité monétaire, semblent plus appropriés. C'est le cas entre autres de l'Empreinte écologique (EE). L'EE « mesure en hectares globaux (pour un individu ou une population) la surface bioproductive nécessaire pour fournir les principales ressources consommées par cette population et pour absorber ses déchets et la compare à la surface effectivement existante » (CASSIERS \& THIRY 2009). En s'inscrivant exclusivement dans le champ des ressources naturelles, l'EE s'oppose fermement aux hypothèses de soutenabilité faible : chaque capital doit être maintenu à des niveaux au moins égaux à ceux nécessaires à assurer les fonctions vitales de la nature à la vie humaine et à des niveaux plus élevés pour assurer un degré raisonnable de résilience environnementale, à savoir la capacité des écosystèmes à se régénérer et se rééquilibrer après un choc (STIGLITZ et al. 2009 ; BOUTAUD \& GONDRAN 2009).

Comme ce bref exemple l'illustre, la même bannière de «soutenabilité » qu'érigent l'ENA et l'EE comporte des visions très différentes du rapport de l'humain à la nature. En termes d'implications normatives et politiques, ces divergences ont des

11 En économie, une externalité est l'effet secondaire d'une activité économique non pris en compte par les prix de marché. La pollution que produit une usine en fabricant des biens est un exemple d'externalité environnementale négative.

12 Hamilton, Atkinson et Pearce (1997), les auteurs de l'ENA, se sont penchés sur la possibilité, pour cet indicateur, de refléter la soutenabilité «forte». Si leur raisonnement semble pertinent d'un point de vue théorique, ils se sont toutefois heurtés à des problèmes de nature empirique, que nous détaillons dans Thiry et Cassiers (2010). 
conséquences non-négligeables notamment sur les contraintes qu'une société perçoit devoir s'imposer afin d'être « soutenable».

\section{Richesse humaine : restreinte à sa productivité et mesurée par les dépenses d'éducation plutôt que par les résultats.}

La manière dont la dimension humaine de la richesse élargie est conçue théoriquement et quantifiée dans l'ENA soulève deux questions. Premièrement, la richesse humaine, nommée «capital humain», est réduite à son potentiel productif (4.1.). Ensuite, refléter le capital humain par les dépenses d'éducation est un choix discutable: se concentrer sur les dépenses d'éducation ne permet pas de saisir les impacts des systèmes éducatifs sur la soutenabilité d'une société (4.2.).

\subsection{Richesse humaine réduite à du potentiel productif}

Dans une conception élargie de la richesse, la dimension humaine de la richesse élargie de l'ENA a pour but d'étendre la définition traditionnelle de ce qui constitue un actif. "There exist additional stocks (...), whose contribution to production is difficult to deny yet which are not considered assets $(.$.$) perhaps the most important of these is$ the knowledge, experience and skills embodied in a nation's populace, its so-called human capital"'(BOLT et al. 2002, p.7).

Contrastant avec cet objectif, le cadre théorique de l'ENA quantifie cette dimension humaine par le «capital humain », conçu comme l'une des diverses composantes d'une fonction de production. En amont de la construction technique de l'ENA, son cadre théorique façonne une perspective productiviste de la richesse humaine, laissant peu d'espace à une approche plus compréhensive de la richesse à transmettre aux générations futures, comme par exemple, la cohésion sociale, le respect, l'altruisme et la culture $^{13}$.

\footnotetext{
${ }^{13}$ En termes de prescriptions normatives, ce productivisme pourrait mener à des propositions restrictives comme en témoigne, par exemple, cette suggestion du parlement européen : "As human capital investments are included in the Genuine Savings, this indicator could support the Lisbon Agenda of the EU of creating a competitive 'knowledge based economy' » (PARLEMENT EuropéEN 2007 : 37). Dans une telle perspective on peut se demander en quoi les études de philosophie ou de littérature seraient considérées comme contribuant à la compétitivité d'une économie de la connaissance et dans quelle mesure ces disciplines bénéficieraient d'investissements en conséquence.
} 
Concevant le capital humain au travers de son potentiel productif uniquement, l'ENA fait de la soutenabilité la transmission d'un potentiel productif plutôt que d'un patrimoine culturel plus large, incluant «les pratiques, représentations, expressions, ainsi que les connaissances et savoir-faire que des communautés, les groupes et, le cas échéant, les individus reconnaissent comme faisant partie de leur patrimoine culturel (...)», que l'UNESCO qualifie de «creuset de la diversité culturelle» dont la préservation est « le garant de la créativité permanente de l’homme » ${ }^{14}$.

\subsection{L'éducation : mesurée par ses dépenses plutôt que par ses résultats}

Considérant que le capital humain dépend grandement du système éducatif, les auteurs de l'ENA évaluent cette dimension à l'aune des dépenses d'éducation. Ce choix de quantification soulève une question importante : cette approche, qui met en exergue les inputs, ne dit rien quant aux résultats ou impacts des systèmes éducatifs. Ainsi par exemple, un accroissement des dépenses en ordinateurs pour les écoles, bien qu'il vise $a$ priori une amélioration de l'accès aux technologies, pourrait contribuer à renforcer la fracture numérique entre les élèves bénéficiant d'un ordinateur à la maison et ceux qui n'en ont pas. En outre, les dépenses d'éducation n'informent pas, comme le voudrait l'ENA, sur l'aptitude du système éducatif à améliorer la productivité.

Se concentrer sur les inputs plutôt que sur les résultats a une portée normative importante. Selon que les dépenses soient investies dans un système éducationnel qui favorise la compétition ou dans un système stimulant plutôt les capacités et les sources d'émancipation personnelles et collectives, la nature de la richesse humaine transmise dans une « société soutenable » s'en trouvera fortement modifiée.

\section{5. Épuisement des ressources naturelles : monétisation contestable et répartition des responsabilités}

L'ENA aborde les variations de richesse naturelle à travers deux dimensions: l'épuisement des ressources naturelles (traité dans cette section) et les dommages liés à la pollution (voire section 6). L'épuisement des ressources naturelles est de loin la dimension la plus discutée dans les littératures écologique et de comptabilité verte. Deux enjeux majeurs sont en débat: la manière dont la nature est monétisée est

\footnotetext{
${ }^{14}$ http:/ / www.unesco.org/culture/ich/index.php?pg=00002
} 
contestable (5.1.) et la manière dont les coûts de l'extraction des ressources sont imputés augmente les inégalités entre pays riches et pays pauvres (5.2).

\subsection{Monétisation contestable de la nature}

Au sein de l'ENA, l'estimation de l'épuisement des ressources naturelles est obtenue en déduisant de l'épargne (telle que comptabilisée en comptabilité nationale) la rente de ces ressources ${ }^{15}$. La rente est la différence entre la valeur de marché de la ressource extraite et son coût moyen d'extraction (NEUMAYER 2000; BOLT et al. 2002)16. Théoriquement, la rente est calculée selon le principe que le prix d'une ressource épuisable augmente de manière autonome au fur et à mesure de son épuisement ${ }^{17}$. Empiriquement toutefois, la valorisation des ressources par les prix de marché n'est pas sans poser de problème.

Premièrement, si les prix de marché sont à même d'évaluer des stocks et des flux en situation de concurrence parfaite (impliquant entre autres une information parfaite), ce n'est plus vrai dans le cas de la nature, dont le marché est soit inexistant soit truffé d'imperfections informationnelles et dont les services comportent de nombreuses externalités. Dans quelle mesure est-il alors pertinent de se fier aux prix de marché pour évaluer la raréfaction des ressources ? Dès lors que les prix n'augmentent pas au fur et à mesure que les ressources s'épuisent, ils ne constituent plus un bon signal d'allocation des ressources. En réponse à cette incapacité des prix de marché à refléter empiriquement l'allocation des ressources, la théorie suggèrerait de créer des «prix comptables ». Ces prix incluraient la valeur des externalités (positives ou négatives). Mais de tels prix nécessitent de modéliser les conséquences de long-terme de l'activité économique sur l'environnement et de l'environnement sur le bien-être (STIGLITZ et al. 2009).

Deuxièmement, la manière dont la rente est calculée participe de manière déterminante à la catégorisation des pays en «soutenables» et "non-soutenables». Plusieurs auteurs ont montré que selon diverses modalités d'évaluation des ressources, les estimations de la soutenabilité d'un pays variaient. Certaines méthodes de calcul impliquent qu'un pays qui dépendrait totalement de l'exploitation de ses ressources,

\footnotetext{
${ }^{15}$ Les ressources renouvelables et non-renouvelables prises en compte dans l'ENA incluent le gaz naturel, l'anthracite, le lignite/la houille, le pétrole, des métaux et minéraux ${ }^{15}$ et les forêts (BOLT et al. 2002).

${ }^{16}$ Dans le cas spécifique des forêts, seule ressource renouvelable prise en compte dans l'ENA, la rente est calculée sur la part de l'extraction qui dépasse l'incrément naturel du volume de bois par année.

17 Voir Thiry et Cassiers (2010) pour le détail de la construction de la rente.
} 
comme l'Arabie-Saoudite par exemple, ne pourrait jamais apparaittre soutenable du point de vue de l'ENA, tandis que d'autres méthodes verraient l'Arabie-saoudite entrer dans la catégorie de soutenabilité (NEUMAYER 2000).

\section{2. Épuisement des ressources : imputations des coûts}

Dans la méthodologie de l'ENA, l'épuisement des ressources non-renouvelables est imputé aux pays extracteurs, le plus souvent pauvres et dont l'extraction est vouée à l'exportation. Ainsi, l'image véhiculée par l'ENA met en exergue la non-soutenabilité des pays exportateurs plutôt que de questionner les schémas de (sur)consommation des pays importateurs ${ }^{18}$.

On peut légitimement être mal à l'aise avec le message qu'adresse l'épargne nette ajustée sur les pays exportateurs de ressources (le pétrole par exemple) : en effet, l'insoutenabilité de leur sentier de croissance n'est imputable qu'à un taux insuffisant de réinvestissement des revenus engendrés par l'exploitation de la ressource naturelle, tandis que la «surconsommation » des pays importateurs n'est pas du tout prise en compte. Les pays développés, qui sont généralement moins bien dotés en ressources naturelles, mais plus riches en capital humain et physique que les pays en développement, apparaissent alors, à tort, comme étant « soutenables». (STIGLITZ et al. 2009, p.76)

À cet égard, selon le Rapport sur les catastrophes dans le monde 2001, contrairement à ce que l'ENA suggère, plusieurs économies avancées opèrent leurs activités à des niveaux non-soutenables. L'EE de ces pays témoigne de l'accumulation d'une dette écologique substantielle ${ }^{19}$.

L'asymétrie de représentation que génère l'ENA en matière de répartition de la charge environnementale a des conséquences non-négligeables dès lors que cet indicateur est amené à jouer un rôle dans la prise de décision, comme l'ont proposé Atkinson et Hamilton (1996) et Hamilton et al. (1997). Ces auteurs avaient suggéré de rendre conditionnelles les aides aux pays en développement qui auraient échoué au «test de soutenabilité faible», en d'autres termes, aux pays ayant une ENA négative. Afin de contrecarrer le problème éthique que soulève ce mode d'imputation des coûts environnementaux, certains indicateurs ont été construits de sorte à imputer le coût de l'épuisement des ressources aux consommateurs, principalement importateurs.

18 Everett \& Wilks (1999), Pillarisetti (2005), FalConi (1999).

19 Fédération internationale des societiés de la Croix-Rouge et du Croissant-Rouge (2001) cité dans PilLarisetTi (2005). 


\section{Dommages liés à la pollution}

Les dommages globaux liés aux émissions de dioxyde de carbone $\left(\mathrm{CO}_{2}\right)$ constituent le dernier élément pris en compte dans l'ENA. Ces données, issues du Carbon Dioxide Analysis Center, sont publiées dans World Bank Development Indicators. «The global marginal social cost of a metric ton of carbon emitted is assumed to $\$ 20$ in 1995 (...) This is deflated for other years using the USA GDP deflator » (BOLT et al. 2002, p.19) ${ }^{20}$.

L'estimation monétaire des dommages du $\mathrm{CO}_{2}$ soulèvent les mêmes problèmes d'incertitude et d'arbitraire que l'évaluation de l'épuisement futur des ressources. «It is debatable whether valuing $\mathrm{CO}_{2}$ emissions with 20 US $\$$ per ton of carbon is sufficient to account for damage caused by greenhouse gases» (NEUMAYER 2000: 273). Cette monétisation est d'autant plus questionnable qu'elle est anthropocentrée : le prix du $\mathrm{CO}_{2}$ est déterminé sur un marché de $\mathrm{CO}_{2}$, dépendant dès lors uniquement de l'offre et la demande humaines de $\mathrm{CO}_{2}$. La difficulté d'évaluer le $\mathrm{CO}_{2}$ par un prix fait l'objet d'une très large littérature, non revue ici. Notre approche spécifique est de montrer que la manière dont le $\mathrm{CO}_{2}$ est pris en compte dans l'ENA soulève un enjeu crucial de responsabilité écologique, au moins à deux égards.

D'abord, la manière dont le $\mathrm{CO}_{2}$ est évalué monétairement, et donc implicitement pondéré par rapport aux autres dimensions de l'ENA, rend cette dimension quasi invisible dans l'indicateur. «En l'état actuel des choses, les prix utilisés pour valoriser les émissions de carbone dans les estimations existantes de l'épargne nette ajustée ne permettent pas de leur faire jouer un rôle significatif dans l'évaluation globale de la durabilité, ce qui sème le doute quant à l'utilité de cet indicateur pour orienter les choix politiques» (STIGLITZ et al. 2009, p.69). Si l'ENA a pour but de signaler le risque de non-soutenabilité d'un pays, le poids relatif des dommages liés au $\mathrm{CO}_{2}$ empêche cet indicateur de montrer du doigt la responsabilité écologique globale de certains pays à fortes émissions de $\mathrm{CO}_{2}$.

Ensuite, l'imputation des émissions de $\mathrm{CO}_{2}$ à un niveau global est discutable en termes de responsabilités. Dans le sillage des accords de Kyoto, les émissions de $\mathrm{CO}_{2}$ sont imputées à la production: si la production est intensivement dédiée à l'exportation, la responsabilité des dommages du $\mathrm{CO}_{2}$ des pays exportateurs est surestimée tandis que la responsabilité des pays importateurs est sous-estimée. Ce choix de construction de l'ENA constitue un biais important en faveur des pays consommateurs de biens produits au prix de fortes émissions de $\mathrm{CO}_{2}$.

Plus fondamentalement, on peut se demander pourquoi la dégradation de l'environnement est seulement prise en compte par le biais des dommages liés aux

\footnotetext{
${ }^{20}$ Les estimations de la pollution atmosphérique des cinq dernières années sont disponibles dans
} Banque Mondiale (2009). 
émissions de $\mathrm{CO}_{2}$. «Les évaluations empiriques de l'épargne nette ajustée souffrent d'un défaut majeur : l'ajustement au titre de la dégradation de l'environnement est limité aux dommages causés par la pollution mondiale liée aux émissions de dioxyde de carbone» (STIGLITZ et al. 2009, p.309). Cette observation amène à élargir l'argument non seulement aux émissions de $\mathrm{CO}_{2}$ mais également à l'épuisement des ressources naturelles : certains facteurs écologiques majeurs sont absents de l'ENA. Certes, les auteurs reconnaissent cette lacune : «the calculations are not comprehensive in that they do not include some important sources of environmental degradation such as underground water depletion, unsustainable fisheries, and soil degradation» (BANQUE Mondiale 2006, p.154). Toutefois, dès lors que l'ENA se présente comme indicateur de soutenabilité, de telles lacunes devraient au moins être explicitement mentionnées et au mieux comblées.

\section{Conclusion}

L'ENA constitue certainement une étape considérable dans la recherche de nouveaux indicateurs de progrès. Le fait que cet indicateur, qui vise un défi global, émane d'une institution globale, accroît son influence potentielle sur la coordination, au niveau international, des politiques nationales de soutenabilité. Plus fondamentalement, cet indicateur prétend élargir le concept de richesse, offrant par là-même une plus grande visibilité aux aspects écologique et humain de la richesse dans la prise de décision.

Toutefois, bien que ces enjeux impératifs soient pris en compte dans l'ENA, la manière dont ils sont comptabilisés réduit considérablement l'étendue des défis considérés et corollairement les réponses à y apporter. L'analyse du cadre théorique de l'ENA a montré que les ressources naturelles, la qualité de l'environnement et le capital humain sont conçus uniquement dans leur dimension productive. Dès lors que les indicateurs ne sont pas de simples reflets passifs d'une réalité mais performent activement cette réalité, l'on peut s'étonner - voire s'inquiéter - de voir l'ENA cloisonnée dans une approche productiviste. Une telle perspective n'offre pas de cadre heuristique pour questionner les raisons systémiques - intrinsèquement liées à l'évolution des systèmes productifs - qui expliquent que les sociétés soient aujourd'hui globalement sur une trajectoire non-soutenable. Si l'ENA illustre la volonté d'enrichir les conceptions traditionnelles de richesse, le cadre théorique dont elle est issue réduit en amont les possibilités d'une redéfinition de la richesse, en portant intrinsèquement dans son axiomatique une vision singulière - productiviste - de ce qu'est la soutenabilité et inextricablement de ce qu'elle devrait être.

Ces fondements normatifs se répercutent et s'amplifient dans les choix méthodologiques sur lesquels l'ENA est construite. L'hypothèse de substituabilité des capitaux, cristallisée dans la somme d'éléments hétérogènes rassemblés sous une même unité de compte, élude la prise en compte des limites physiques de l'écosystème, à un moment où le respect de celles-ci constitue un impératif à l'aune duquel les sociétés 
doivent accommoder leurs modes de vie. À la lumière de l'ENA, tant que le niveau d'investissement en capital humain ou produit est suffisant pour compenser une perte de ressources épuisables ou une dégradation de l'environnement, l'économie se maintient sur une trajectoire soutenable. Par ailleurs, considérant du «capital humain » plutôt que de la «richesse humaine", l'ENA restreint les êtres humains à leur productivité, ne laissant aucun espace aux sources «économiquement improductives » de richesses humaines (institutions démocratiques, altruisme, cohésion sociale, respect, etc.). En outre, opérationnaliser le «capital humain» par les dépenses d'éducation ne permet pas de juger les résultats des systèmes éducatifs. Concernant les ressources naturelles, leur évaluation en termes monétaires a été justifiée par des contraintes d'ordre pratique et/ou méthodologique. Ces motifs ont subrepticement évincé de la réflexion des questions plus fondamentales : dans quelle mesure les prix sont-ils un bon signal d'allocation des ressources? Sont-ils capables de refléter la valeur de la nature indépendamment des biens et services qu'elle fournit? Les prix de marché sont-ils pertinents pour pondérer implicitement les différentes dimensions de l'ENA entre elles? De plus, le fait que les coûts de l'extraction des ressources soient imputés aux pays extracteurs n'est pas sans conséquence : la charge environnementale pèse sur les épaules des pays exportateurs de ressources (pauvres, pour la plupart), plutôt que sur celles des pays qui les consomment (principalement des pays riches), dont la surconsommation est passée sous silence. De tels choix méthodologiques peuvent s'avérer cruciaux dans le cas où, par exemple, l'ENA servirait à l'élaboration de critères d'accès à de l'aide financière.

La décomposition de l'ENA a montré comment une mesure, qu'un survol rapide donne à percevoir a priori comme neutre, est implicitement porteuse de valeurs et de visions du monde. Par son mode de construction et le modèle théorique qui y préside, elle crée ses propres catégories, distinguant les pays «soutenables» des «nonsoutenables». Ces catégories influencent considérablement la réalité que prétend refléter l'indicateur ainsi que les décisions qui en sont issues. Cette portée normative n'est pas l'apanage exclusif de l'ENA et est inhérente à tout indicateur. S’il est acquis aujourd'hui que ce que nous mesurons influence nos actions, nous avons montré ici que la manière dont nous mesurons est tout aussi cruciale. C'est pourquoi, à un moment où de très nombreux nouveaux indicateurs de "progrès » (dans ses aspects écologique et humain) sont mis en débat, à un moment où les systèmes de décisions se fondent de plus en plus largement sur des indicateurs, une lecture critique des fondements et impacts normatifs inhérents aux choix de mesures s'avère capitale. Cet exercice devrait s'appliquer au plus grand nombre des outils de mesures proposés aujourd'hui. Un défi démocratique est en jeu : inviter à débattre des indicateurs, comme c'est le cas aujourd'hui, sans expliciter les valeurs implicites inhérentes à leur mode de construction, contribuerait à duper les participants aux débats quant à leur réel pouvoir de délibération. 


\section{Bibliographie}

Banque Mondiale (2006): Where is the Wealth of Nations? Measuring Capital for the 21th Century. Washington D.C.: The World Bank.

Banque Mondiale (2009): World Development Indicators 2009. Washington D.C.: The World Bank.

Bolt K.., Matete M., Clemens M. (2002): Manual for Calculating Adjusted Net Savings. Washington D.C.: The World Bank Environment Department.

Boutaud A., Gondran N. (2009): L'empreinte écologique. Paris: La Découverte.

CAssiers I., Thiry G. (2009): «Au-delà du PIB: réconcilier ce qui compte et ce que l'on compte ». In Regards Economiques, 75, pp.1-15.

Daly H., CoBB J. (1989): For the common good. Boston: Beacon Press.

Dasgupta P., Heal G. (1979): Economic Theory and Exhaustible Resources. Cambridge: Cambridge University Press.

DESROSIERES A. (2008): Pour une sociologie historique de la quantification. L'argument statistique I. Paris: Presses de l'École des Mines.

European Parliament (2007): Alternative Progress Indicators to Gross Domestic Product $(G D P)$ as a Means towards Sustainable Development. Study requested by the EU Parliament's Committee on the Environment, Public Health and Food Safety.

EveretT G., Wilks A. (1999): «The World Bank's Genuine Savings Indicator : a Useful Measure of Sustainability?. In Bretton Woods Project Working Paper, October 1999, pp.1-10.

FALCONI F. (1999): «Indicadores de sustentabilidad debil, un palido reflejo de una realidad mas robusta y compleja ». In Ecologia politica, vol.18, pp.65-99.

FERREIRA S., VINCENT J. (2005): «Genuine Savings: Leading Indicator of Sustainable Development? ». In Economic Development and Cultural Change, vol.53 n³, pp.737-54.

FOURQUeT F. (1980): Les comptes de la puissance. Histoire de la comptabilité nationale. Paris: Éditions Encres.

GADREy J., JANY-CATRICE F. (2007): Les nouveaux indicateurs de richesse. Paris: La Découverte - Repères.

Hamilton K. (1993): «Greening the human development index ». Document non publié.

Hamilton K. (1994): «Green Adjustment to GDP” ». In Resources Policy, vol.20 n³, pp.155-168. 
Hamilton K., Atkinson G., Pearce D. (1997): « Genuine Savings as an Indicator of Sustainability ». In CSERGE Working Paper, n³, pp.1-28.

Hamilton K., Clemens M. (1999): " Genuine Savings Rates in Developing Countries ». In The World Bank Economic Review, vol.13 n², pp.333-356.

HARTWICK J. (1990): «National resources, national accounting, and economic depreciation’ ». In Journal of Public Economics, vol.43 n³, pp.291-304.

JACKSON T.(2009): Prosperity without Growth. Economics for a Finite Planet. New-York: Earthscan.

Neumayer E. (2000): "Resource Accounting in Measure of Unsustainability. Challenging the World Bank's Conclusions». In Environmental and Resource Economics, vol.15, pp.257-278.

PeArCe D.W., AtKinson G. (1993): «Capital theory and the measurement of sustainable development: an indicator of weak sustainability». In Ecological Economics, vol.8, pp.103-108.

Pearce D.W., Hamilton K., AtKinson G. (1996): «Measuring Sustainable Development: Progress on Indicators ». In Environment and Development Economics, vol. $1 \mathrm{n}^{\circ} 1$, pp.85-101.

PEZZEY J. (1989): «Economic Analysis of Sustainable Growth and Sustainable Development ». In World Bank Working Paper, $\mathrm{n}^{\circ} 15$.

PILLARISETTI J.R. (2005): «The World Bank's 'genuine savings' measure and sustainability ». In Ecological Economics, vol.55, pp.599-609.

PillarisetTi J.R., VAN DEN Bergh J. (2008): «Sustainable Nations : What do Aggregate Indicators Tell Us?». In Tinbergen Institute Discussion Paper, $\mathrm{n}^{\circ}$ 012/3.

STIGlitz J., SEN A., Fitoussi J.-P. (2009): Report of the Commission on the Measurement of Economic performance and Social Progress. Document online, disponible sur http://www.stiglitz-sen-fitoussi.fr/documents/rapport_francais.pfd

TAlberth J., CobB C. et SlatTery N. (2006): The Genuine Progress Indicator 2006. A tool for Sustainable Development. Oakland: Redefining Progress.

THIRY G., CASSIERS I. (à paraitre): «Alternative Indicators to GDP: Values behind Numbers. Adjusted Net Savings in Question ». In Applied Research in Quality of Life.

ThIRY G., CASSIERS I. (2010): «Alternative Indicators to GDP: Values behind Numbers. Adjusted Net Savings in Question ». In IRES Discussion Paper, n¹8.

United Nations General Assembly - World COMmission on ENVIRONMENT And Development (1987): Our Common Future. Online document. 
United NAtions Statistical Department (1993): Integrated Environmental and Economic Accounts: Interim Version. New York: United Nations. 